\title{
Spontaneous Breaking of Isotropy Observed in the Electronic Transport of Rare-Earth Tritellurides
}

\author{
A. A. Sinchenko, ${ }^{1,2, *}$ P. D. Grigoriev, ${ }^{3}$ P. Lejay, ${ }^{2}$ and P. Monceau ${ }^{2}$ \\ ${ }^{1}$ Kotelnikov Institute of Radioengineering and Electronics of RAS, Mokhovaya 11-7, 125009 Moscow, Russia \\ ${ }^{2}$ Universite Grenoble Alpes, Institut Neel, F-38042 Grenoble, France; CNRS, Institut Neel, F-38042 Grenoble, France \\ ${ }^{3}$ L.D. Landau Institute for Theoretical Physics, 142432 Chernogolovka, Russia \\ (Received 19 June 2013; revised manuscript received 25 October 2013; published 24 January 2014)
}

\begin{abstract}
We show that the isotropic conductivity in the normal state of rare-earth tritelluride $R \mathrm{Te}_{3}$ compounds is broken by the occurrence of the unidirectional charge density wave $(\mathrm{CDW})$ in the $(a, c)$ plane below the Peierls transition temperature. In contrast with quasi-one-dimensional systems, the resistivity anomaly associated with the CDW transition is strong in the direction perpendicular to the CDW wave vector $\mathbf{Q}$ ( $a$ axis) and very weak in the CDW wave vector $\mathbf{Q}$ direction ( $c$ axis). We qualitatively explain this result by calculating the electrical conductivity for the electron dispersion with momentum-dependent CDW gap as determined by angle-resolved photoemission spectroscopy. Similar measurements of in-plane conductivity may uncover the gap anisotropy in other compounds for which angle-resolved photoemission spectroscopy is not available.
\end{abstract}

DOI: 10.1103/PhysRevLett.112.036601

PACS numbers: 72.15.Nj, 71.45.Lr, 61.44.Fw

Quasi-two-dimensional (quasi-2D) systems with strong electronic correlations exhibit a wide variety of properties due to strong coupling and competition among charge, spin, orbital, and lattice degrees of freedom. One fingerprint of the resulting ground states is often the formation of unidirectional charge or spin modulations, as continuously investigated in manganites [1], organic compounds [2], and cuprates $[3,4]$. There is a large debate to associate or not the high-temperature superconductivity in the latter compounds with the existence of charge modulation.

Very recently, a new family of quasi-2D compounds, namely rare-earth tritellurides $R \mathrm{Te}_{3}(R=\mathrm{Y}, \mathrm{La}, \mathrm{Ce}, \mathrm{Nd}$, $\mathrm{Sm}, \mathrm{Gd}, \mathrm{Tb}, \mathrm{Ho}, \mathrm{Dy}, \mathrm{Er}, \mathrm{Tm})$ has raised intense research activity [5-7]. These layered compounds have a weakly orthorhombic crystal structure (space group $\mathrm{Cmcm}$ ). They are formed of double layers of nominally square-planar Te sheets, separated by corrugated $R T$ e slabs. In this space group, the long $b$ axis is perpendicular to the Te planes. These systems exhibit an incommensurate charge density wave (CDW) through the whole $R$ series [7,8], with a wave vector $\mathbf{Q}_{\mathrm{CDW} 1}=\left(0,0, \sim 2 / 7 c^{*}\right)$ and a Peierls transition temperature above $300 \mathrm{~K}$ for the light atoms $(\mathrm{La}, \mathrm{Ce}$, $\mathrm{Nd}$ ). For the heavier $R$ (Tb, Dy, Ho, Er, Tm) a second CDW occurs at low temperature with the wave vector $\mathbf{Q}_{\mathrm{CDW} 2}=$ $\left(\sim 2 / 7 a^{*}, 0,0\right)$ perpendicular to $\mathbf{Q}_{\mathrm{CDW}}$. The $R \mathrm{Te}_{3}$ family can be considered as a model system in which the structure of the CDW ground state can be theoretically studied [9]. Thus, a phase diagram as a function of the electron-phonon parameter was derived with a bidirectional (checkerboard) state if the CDW transition temperature is sufficiently low, whereas a unidirectional stripe state, as observed experimentally, occurs when the transition temperature is higher. This result is relevant for a deeper understanding of the charge pattern in highly correlated materials, and particularly to the recent determination of the biaxial CDW in underdoped cuprates [10].

Below the Peierls transition, in all $R \mathrm{Te}_{3}$ compounds, the Fermi surface (FS) is partially gapped resulting in a metallic behavior at low temperature. The layered $R \mathrm{Te}_{3}$ compounds exhibit a large anisotropy between the resistivity along the $b$ axis and that in the $(a, c)$ plane, typically $\sim 40$ below $T_{\mathrm{CDW} 1}$ and much higher at low temperature [11]. The effect of the CDW on the in-plane resistivity observed in experiments was very weak, no more than a few percent of the total resistance [7,11]. However, due to the unidirectional character of the CDW $[8,9,12]$, a conductivity anisotropy in the $(a, c)$ plane should be expected in the CDW state. In the present Letter, we report the first observation and theoretical analysis of the in-plane conductivity anisotropy in $R \mathrm{Te}_{3}$ compounds below the Peierls transition.

We have studied $\mathrm{TbTe}_{3}, \mathrm{DyTe}_{3}$, and $\mathrm{HoTe}_{3}$ compounds. In $\mathrm{DyTe}_{3}$ the upper CDW appears just at room temperature at $T_{\mathrm{CDW} 1}=302 \mathrm{~K}$ and the lower $\mathrm{CDW}$ at $T_{\mathrm{CDW} 2}=49 \mathrm{~K}$. In $\mathrm{HoTe}_{3}$ the first and the second CDW transitions take place at $T_{\mathrm{CDW} 1}=283 \mathrm{~K}$ and $T_{\mathrm{CDW} 2}=110 \mathrm{~K}$, correspondingly [7]. In $\mathrm{TbTe}_{3}$, the CDW ordering is observed well above room temperature $\left(T_{\mathrm{CDW} 1}=336 \mathrm{~K}\right)$. The second CDW transition in this compound was recently reported $T_{\mathrm{CDW} 2}=41 \mathrm{~K}$ [13], the lowest in the $R \mathrm{Te}_{3}$ series.

Single crystals of $R \mathrm{Te}_{3}$ were grown by a self-flux technique under purified argon atmosphere as described previously [14]. Thin single-crystal samples with a square shape and with a thickness less than $1 \mu \mathrm{m}$ were prepared by micromechanical exfoliation of relatively thick crystals glued on a sapphire substrate. The quality of selected crystals and the spatial arrangement of crystallographic 
axes were controlled by $x$-ray diffraction. The superlattice spots for the upper CDW were clearly observed along the $c$ axis, demonstrating CDW ordering just in this direction. Conductivity anisotropy measurements were performed using the Montgomery technique [15,16].

Figure 1 shows the temperature dependence of resistivities of $\mathrm{TbTe}_{3}, \mathrm{DyTe}_{3}$, and $\mathrm{HoTe}_{3}$ measured (with the help of the Montgomery method) along the $c$ and $a$ axes together with the anisotropy ratio $\rho_{a} / \rho_{c}$ in the $a c$ plane. As can be seen, above the Peierls transition temperature $T_{\mathrm{CDW} 1}$, all studied compounds are practically isotropic in the $a c$ plane and $\rho_{a} / \rho_{c} \approx 1$. Below $T_{\mathrm{CDW} 1}$, the ratio $\rho_{a} / \rho_{c}$ strongly increases and reaches $\sim 1.4$ for $\mathrm{TbTe}_{3}$ and $\sim 2$ for $\mathrm{DyTe}_{3}$ and $\mathrm{HoTe}_{3}$ at low temperature. The observed effect of the CDW on the resistivity along the $c$ axis is much weaker than the change of resistivity along the $a$ axis. Thus, the transition into the CDW state in rare-earth tritellurides leads to a stronger increase of resistance in the direction perpendicular to the CDW $\mathbf{Q}$-vector than in the direction parallel to the CDW Q-vector. Such behavior is inverse to that observed in quasi-one-dimensional (quasi-1D) compounds with a CDW where the anisotropy is considerable in the normal state and strongly decreases below the Peierls transition [17].

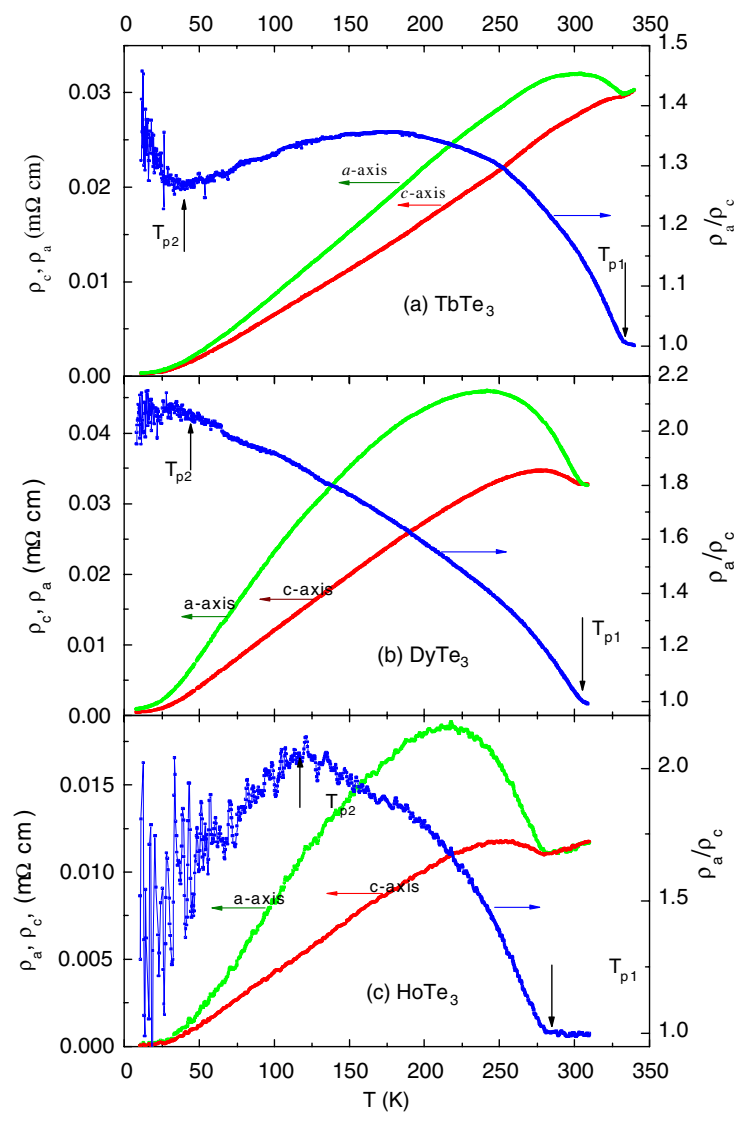

FIG. 1 (color online). Temperature dependence of resistivity of (a) $\mathrm{TbTe}_{3}$, (b) $\mathrm{DyTe}_{3}$, and (c) $\mathrm{HoTe}_{3}$ along the $a$ and the $c$ axis directions and conductivity anisotropy, $\rho_{a} / \rho_{c}$, in the $a-c$ plane.
In $\mathrm{DyTe}_{3}$ and $\mathrm{HoTe}_{3}$ the observed anisotropy starts to decrease below $T=T_{\mathrm{CDW} 2}$. On the contrary, in $\mathrm{TbTe}_{3}$ (see Fig. 1) one observes a sharp increase of anisotropy below $T=41 \mathrm{~K}$, which coincides well with $T_{\mathrm{CDW} 2}$ in this compound as reported in Ref. [13].

To understand the anisotropic influence of the CDW on the resistivity along the $a$ and $c$ axes, shown in Fig. 1, we calculate the electron conductivity $\sigma_{x x}=1 / \rho_{a}$ and $\sigma_{y y}=1 / \rho_{c}$ in the CDW state as a function of temperature. In the $\tau$ approximation, the conductivity along the main axes is given by [18]

$$
\sigma_{i}(T)=2 e^{2} \tau \sum_{\boldsymbol{k}} v_{i}^{2}(\boldsymbol{k})\left\{-n_{F}^{\prime}[\epsilon(\boldsymbol{k})]\right\},
$$

where $e$ is the electron charge, $\tau$ is the mean free time, $\boldsymbol{k}$ is electron momentum [19], $v_{i}$ is the component of the electron velocity along the $i$ direction, $n_{F}^{\prime}(\varepsilon)=-1$ / $\left\{4 T \cosh ^{2}[(\varepsilon-\mu) / 2 T]\right\}$ is the derivative of the Fermi distribution function, which restricts the summation over momentum to the vicinity of FS, $\mu$ is the chemical potential, and $\varepsilon(\boldsymbol{k})$ is the electron dispersion. The factor 2 in Eq. (1) comes from the spin degeneracy.

The momentum dependence of electron velocities $v_{x}$ and $v_{y}$ can be obtained from electron dispersion with and without the CDW gap. Without CDW, the in-plane electron dispersion in $R \mathrm{Te}_{3}$ is described by a simple $2 \mathrm{D}$ tight binding model of the Te plane as developed in [6] in which the square net of $\mathrm{Te}$ atoms forms perpendicular chains created by the in-plane $p_{x}$ and $p_{z}$ orbitals. The model parameters consist of an electron hopping term along a particular chain, $t_{\|}$, and perpendicular to the chain, $t_{\perp}$. Fermi surface curvature is proportional to $t_{\perp} / t_{\|}$

$$
\begin{aligned}
\epsilon_{1}\left(k_{x}, k_{y}\right)= & -2 t_{\|} \cos \left[\left(k_{x}+k_{y}\right) a / 2\right] \\
& -2 t_{\perp} \cos \left[\left(k_{x}-k_{y}\right) a / 2\right]-E_{F}, \\
\epsilon_{2}\left(k_{x}, k_{y}\right)= & -2 t_{\|} \cos \left[\left(k_{x}-k_{y}\right) a / 2\right] \\
& -2 t_{\perp} \cos \left[\left(k_{x}+k_{y}\right) a / 2\right]-E_{F},
\end{aligned}
$$

where the calculated parameters for $\mathrm{TbTe}_{3}$ are $t_{\|} \approx 2 \mathrm{eV}$, $t_{\perp} \approx 0.37 \mathrm{eV}$, and $a \approx 4.4 \AA[6,20]$. These parameters slightly differ for other compounds of this family. The Fermi energy $E_{F} \approx 1.48 \mathrm{eV}$ is chosen to fit the angle-resolved photoemission spectroscopy (ARPES) data on the FS in $\mathrm{TbTe}_{3}[6,21]$. The FS calculated above the CDW transition from Eq. 2 is shown in Fig. 2 (inset). At $t_{\perp}=0$ the FS contains only straight lines $k_{x} \pm k_{y}=$ const $=$ $\pm(2 / a) \arccos \left(E_{F} / 2 t_{\|}\right)$, which warp at nonzero $t_{\perp}$.

The electron velocity, calculated from Eq. (2) using

$$
v_{x}=\partial \varepsilon / \partial k_{x}, \quad v_{y}=\partial \varepsilon / \partial k_{y},
$$

varies considerably along the Fermi surface. The $k_{x}$ dependence of $v_{x}^{2}$ and $v_{y}^{2}$ on the FS above the CDW transition is shown in Fig. 2. One can see that the maxima 


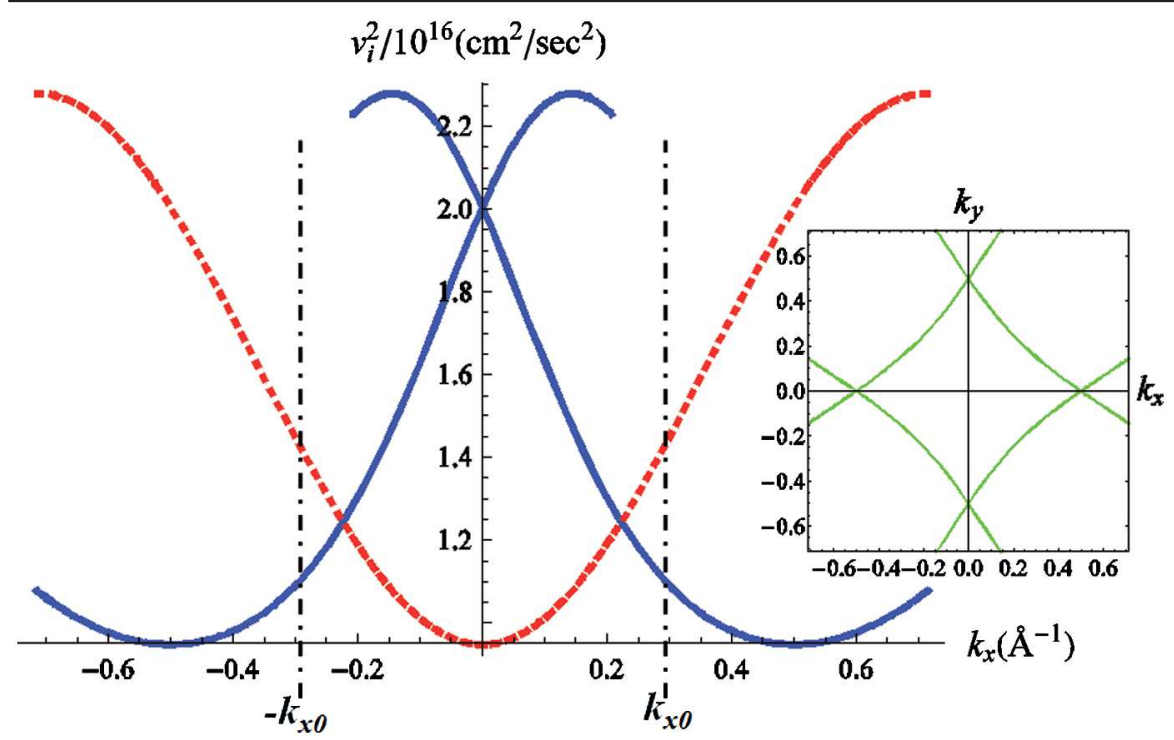

FIG. 2 (color online). Variation of two main components $v_{x}^{2}$ (solid blue line) and $v_{y}^{2}$ (dashed red line) of the electron velocity along the Fermi surface for $\mathrm{TbTe}_{3}$ above the CDW transition, as calculated from Eqs. (2) and (3) with parameters: $t_{\|}=2 \mathrm{eV}, t_{\perp}=0.37 \mathrm{eV}$. Inset: Fermi surface of $\mathrm{TbTe}_{3}$ above the CDW transition (green solid line) calculated from Eq. (2). In the CDW state, the gap covers the region $\left|k_{x}\right| \leq k_{x 0} \approx 0.29 \AA^{-1}$, as is shown by vertical dashed-dotted lines, and violates the equivalence between $v_{x}^{2}$ and $v_{y}^{2}: v_{x}^{2}$ is maximum in the region under the CDW gap while $v_{y}^{2}$ is maximum in the ungapped region.

of $v_{x}^{2}$ and $v_{y}^{2}$ are on different parts of the FS. This asymmetry comes from finite $t_{\perp}$. Without CDW, the summation over momentum in Eq. (1) gives the same result for $v_{x}^{2}$ and $v_{y}^{2}$, which leads to an isotropic electron conductivity. The CDW gap violates this balance because it covers only some parts of FS. The $k_{x}$ dependence of the CDW gap in $\mathrm{TbTe}_{3}$ is shown in Fig. 13 of Ref. [6]. The CDW gap is nonzero only in the region $\left|k_{x} / a^{*}\right| \equiv$ $\left|k_{x} a / 2 \pi\right| \leq 0.2$, which corresponds to $\left|k_{x}\right| \leq k_{x 0} \approx 0.29 \AA^{-1}$. As one can see in Fig. $2, v_{x}^{2}$ has a maximum value just in this region under the CDW gap, while $v_{y}^{2}$ is maximum in the ungapped region $\left|k_{x}\right|>k_{x 0}$. At low temperature $T \ll \Delta_{\mathrm{CDW}}$, the integration over momentum in Eq. (1) includes only the ungapped FS parts and gives the following anisotropy ratio [22]:

$$
\frac{\sigma_{y y}}{\sigma_{x x}} \approx \frac{\int_{\left|k_{x}\right| \geq k_{x 0}} d k_{x} \sqrt{1+\left|\frac{d k_{y}}{d k_{x}}\right|_{F S}^{2}} v_{y}^{2}}{\int_{\left|k_{x}\right| \geq k_{x 0}} d k_{x} \sqrt{1+\left|\frac{d k_{y}}{d k_{x}}\right|_{F S}^{2}} v_{x}^{2}} .
$$

Taking the same parameters as for Eq. (2) and Ref. [6], one gets $\left(\sigma_{y y} / \sigma_{x x}\right) \approx 1.96$. Equation (4) means that the resistivity ratio $\rho_{a} / \rho_{c} \approx \sigma_{y y} / \sigma_{x x}$ increases from 1 to almost 2 as the temperature decreases below $T_{\mathrm{CDW} 1}$. This agrees well with the experimental data in Fig. 1. The anisotropy ratio in Eq. (4) depends strongly on the value of $t_{\perp}$. In Fig. 3, we plot this anisotropy ratio as a function of $t_{\perp}$ with arrows corresponding to the experimental values for $\mathrm{TbTe}_{3}$, $\mathrm{HoTe}_{3}$, and $\mathrm{DyTe}_{3}$.

From the calculated momentum dependence of the velocity squared (see Fig. 2) and from the sign of resistivity anisotropy one can determine that the regions $\left|k_{x}\right|<k_{x 0}$ rather than $\left|k_{x}\right|>k_{x 0}$ are covered by the CDW gap even without ARPES data on the momentum dependence of CDW energy gap. The value of the resistivity anisotropy can even give a rough estimate of $k_{x 0}$. Similar analysis may provide some information on the momentum dependence of the CDW energy gap in the compounds, where ARPES data in the CDW state are not available.

To calculate the temperature dependence of the resistivity anisotropy, one needs a detailed knowledge of the temperature evolution of the CDW gap $\Delta(T, \mathbf{k})$. The momentum dependence of the CDW gap at the Fermi level

$$
\Delta(T, \boldsymbol{k}) \approx \Delta_{0}(T) \Delta(\boldsymbol{k}) \approx \Delta_{0}(T)\left(1-k_{x}^{2} / k_{x 0}^{2}\right),
$$

is taken as a simplest fit of the experimental data in Fig. 13 of Ref. [6]. Equation (5) implies that as the temperature decreases, only the amplitude $\Delta_{0}(T)$ but not the momentum dependence of $\Delta(\boldsymbol{k})$ changes, which reasonably agrees with ARPES data. Let us take the growth of $\Delta_{0}(T)$ at the transition temperature $T_{\mathrm{CDW} 1}$ as

$$
\Delta_{0}(T) \approx \Delta_{0}\left(1-T^{2} / T_{\mathrm{CDW} 1}^{2}\right)^{\alpha} .
$$

The new electron dispersion is given by [23]

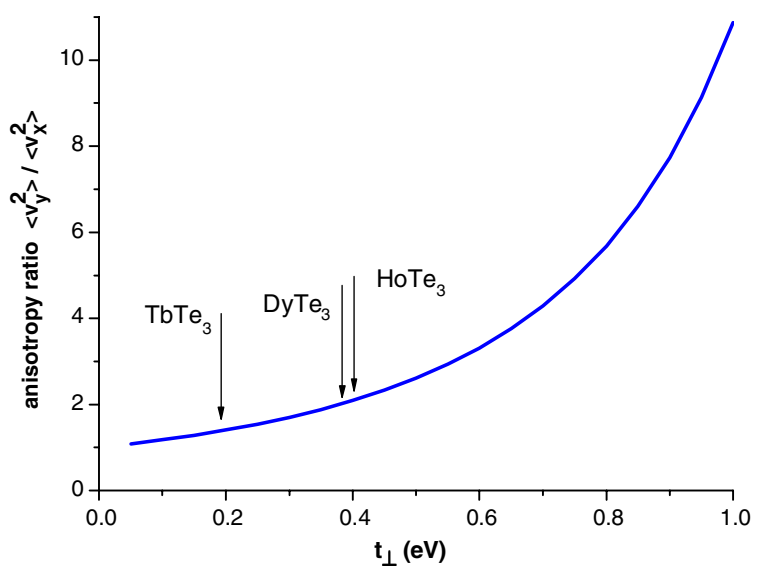

FIG. 3 (color online). The anisotropy ratio of the resistivity along the $a$ and $c$ axes $\rho_{a} / \rho_{c} \approx\left\langle v_{y}^{2}\right\rangle_{\mathrm{FS}} /\left\langle v_{x}^{2}\right\rangle_{\mathrm{FS}}$ as a function of $t_{\perp}$ calculated from Eqs. (2)-(4) for $t_{\|}=2 \mathrm{eV}$. The arrows indicate the experimental anisotropy ratio for $\mathrm{TbTe}_{3}, \mathrm{DyTe}_{3}$, and $\mathrm{HoTe}_{3}$. 


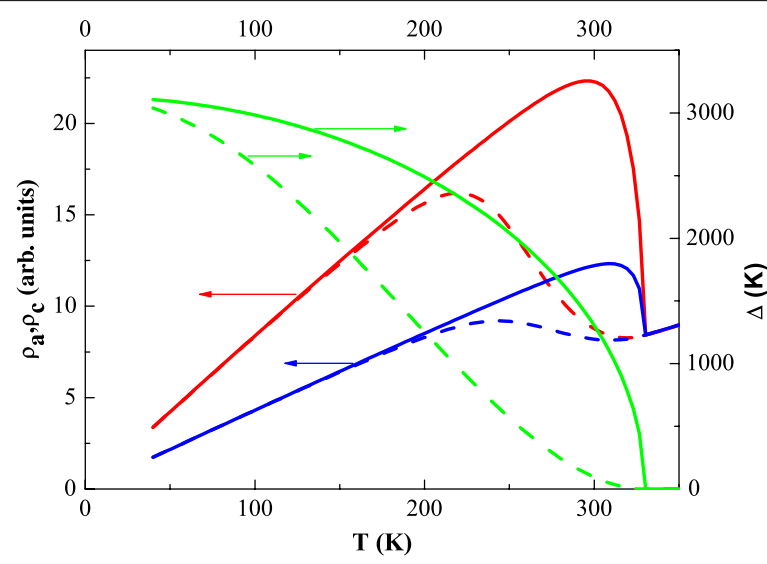

FIG. 4 (color online). The calculated temperature dependence of resistivity $\rho_{a}=1 / \sigma_{x x}$ solid (dashed) red lines and $\rho_{c}=1 / \sigma_{y y}$ solid (dashed) blue lines in the presence of the CDW gap, $\Delta$, with the momentum dependence given by Eq. (5), with the mean-field temperature dependence $\alpha=1 / 2$ in Eq. (6) (solid green line), and for the weaker temperature dependence near $T_{\mathrm{CDW} 1}$ for $\alpha=2$ (dashed green line). The parameters for calculations are those for $\mathrm{TbTe}_{3}$ with $t_{\|}=2 \mathrm{eV}, t_{\perp}=0.37 \mathrm{eV}$, $\Delta_{0}=0.27 \mathrm{eV}, k_{x 0}=0.29 \AA^{-1}$.

$$
E(\boldsymbol{k})=\sqrt{\varepsilon^{2}(\boldsymbol{k})+\Delta^{2}(T, \boldsymbol{k})} .
$$

Since the $\boldsymbol{k}$ dependence of $\varepsilon(\boldsymbol{k})$ is much stronger than that of $\Delta_{0}(\boldsymbol{k})$, the electron velocity in the presence of the CDW gap is

$$
v_{i \Delta}(\boldsymbol{k})=\frac{\partial E(\boldsymbol{k})}{\partial k_{i}} \approx \frac{\varepsilon(\boldsymbol{k}) \partial \varepsilon(\boldsymbol{k}) / \partial k_{i}}{\sqrt{\varepsilon^{2}(\boldsymbol{k})+\Delta^{2}(T, \boldsymbol{k})}}=v_{i}(\boldsymbol{k}) \frac{\varepsilon(\boldsymbol{k})}{E(\boldsymbol{k})} .
$$

Substituting this new electron velocity to Eq. (1), we obtain

$$
\begin{aligned}
\sigma_{i}(T)= & \frac{e^{2} \rho_{F} \tau}{d} \int_{-\pi / a}^{\pi / a} \frac{a d k_{x}}{2 \pi} \sqrt{1+\left(\frac{d k_{y}}{d k_{x}}\right)^{2}} v_{i}^{2}\left(k_{x}\right) \\
& \times \int \frac{d \varepsilon-n_{F}^{\prime}\left[\sqrt{\varepsilon^{2}+\Delta^{2}\left(T, k_{x}\right)}\right] \varepsilon^{2}}{v_{F}},
\end{aligned}
$$

where $\Delta=\Delta\left(T, k_{x}\right)$ is given by Eqs. (5)-(6), and $v_{i}\left(k_{x}\right)$ is given by Eq. (3) with the ungapped electron dispersion (2).

First, we take $\alpha=1 / 2$ as in the mean-field approximation. The numerical integration of Eq. (9) using (5)-(8) gives the temperature dependence of the in-plane resistivity along the $a$ and $c$ axes shown in Fig. 4 by a solid blue line for $\rho_{c}$ and by a solid red line for $\rho_{a}$, with the parameters corresponding to $\mathrm{TbTe}_{3}$, the same as used for Fig. 2.

The comparison between experimental data [Fig. 1(a)] and calculated resistivity shown in Fig. 4 for $\mathrm{TbTe}_{3}$ indicates that the increase of resistivity along both the $a$ and $c$ axes is much more smooth than that derived from the mean field temperature dependence of the CDW gap. Fluctuations may yield a weaker growth of $\Delta_{0}(T)$ near $T_{\mathrm{CDW} 1}$ with the exponent $\alpha$ in Eq. (6) higher than $1 / 2$. This dependence is represented in Fig. 4 for $\alpha=2$ by a dashed green line (to be compared with the solid green line for the mean field prediction). The temperature dependence of the calculated resistivity along the $c$ and $a$ axes are drawn in Fig. 4 with a dashed blue line for $\rho_{c}$ and a dashed red line for $\rho_{a}$, showing a better qualitative agreement with experimental data.

Measurements of the $T$ dependence of the CDW gap for $R \mathrm{Te}_{3}$ compounds are scarce. Detailed measurements by ARPES on $\mathrm{ErTe}_{3}$ suggest a mean-field type behavior but, as noted by the authors of Ref. [24], somewhat suppressed from the mean-field curve. Study of the collective modes in $\mathrm{DyTe}_{3}$ and $\mathrm{LaTe}_{3}$ obtained by Raman scattering has shown that the amplitude CDW mode develops a succession of two mean-field BCS-like transitions with different critical temperatures ascribed to the presence of two adjacent $\mathrm{Te}$ planes in the crystal structure [8]. The occurrence of these two transitions with fluctuating effects between them may yield the soft growth extended in temperature of $\Delta(T)$.

A second CDW phase transition appears at $T_{\mathrm{CDW} 2}<$ $T_{\mathrm{CDW} 1}$. This new transition may modify the momentum dependence of the upper CDW and reduce the resistivity anisotropy as experimentally measured in $\mathrm{DyTe}_{3}$ [Fig. 1(b)] and $\mathrm{HoTe}_{3}$ [Fig. 1(c)]. The increase of anisotropy below $T_{\mathrm{CDW} 2}=41 \mathrm{~K}$ in $\mathrm{TbTe}_{3}$ may indicate a specific property of the low-T CDW state in this compound, for instance, a possible interference between the two CDW distortions.

In spite of the drastic modification in the FS topology occurring at $T_{\mathrm{CDW} 1}$ and $T_{\mathrm{CDW} 2}$ as determined by ARPES [6], a relatively small effect is induced on resistivity as seen in Fig. 1. The density of states (DOS) at the Fermi level was estimated to be suppressed in the CDW state to $77 \%$ of the value in the nonmodified state $[6,25]$, and the area of the FS gapped by the first CDW is 3 times the area gapped by the second one $[24,25]$. The $\mathrm{CDW}$ transition at $T_{\mathrm{CDW} 2}$ is barely visible in transport measurements (see Fig. 1), indicating a weak change in the product of the DOS and the mean square velocity of the Fermi level induced by the CDW transition. One possible reason is a modification of the electron dispersion [see Eq. (7) and Ref. [23]] and, hence, of the quasiparticle effective mass and velocity in the ungapped parts of the FS as compared to those in the metallic state. This renormalization of electron dispersion may partially compensate the opening of the CDW gap on some FS part, similarly to that calculated for the quasi-1D dispersion in Ref. [26].

The magnitude of the CDW gaps in $R \mathrm{Te}_{3}$ compounds are $\Delta_{0} \sim 260-400 \mathrm{meV}$ leading to mean-field (MF) transition temperatures $T_{\mathrm{MF}}$ in the range $1500-2000 \mathrm{~K}$, while the upper CDW transition occurs between 260 and $400 \mathrm{~K}$, leading to a large ratio $2 \Delta(0) / k_{B} T_{\mathrm{CDW}} \sim 10-15$ higher than 3.52 , the BCS value. In the conventional weak coupling FröhlichPeierls model, nesting of pieces of the FS yields the divergence of the electronic susceptibility with the concomitant Kohn anomaly in the phonon spectrum at the same wave vector $2 k_{F}$. However, very few CDW systems follow this model. Similarly, large $2 \Delta(0) / k_{B} T_{c}$ in transition metal dichalcogenides $M X_{2}$ [27] and in one-dimensional systems [28] were observed. For the latter compounds, strong 
fluctuations were believed to reduce the critical CDW temperature below $T_{\mathrm{MF}}$. For $M X_{2}$ compounds, namely $2 \mathrm{H}-\mathrm{TaSe}_{2}$, McMillan reformulated the microscopic CDW theory in considering that phonons over a substantial part of the Brillouin zone (BZ) soften through the transition and that the lattice entropy is much larger than the electronic entropy [29]. While nesting between large parts of the FS in $R \mathrm{Te}_{3}$ compounds is clearly seen from ARPES measurements [6], the role of the strong electron-phonon coupling, and essentially its wave vector dependence has been recently put into evidence to determine the selection of the order parameter in $\mathrm{ErTe}_{3}$ from Raman scattering [30]. However, inelastic neutron or x-ray scattering experiments are clearly needed for phonon spectra, especially for the observation of phonon softening in the BZ.

In conclusion, we have shown that the quasi-isotropic conductivity in the normal state of untwinned $R \mathrm{Te}_{3}$ compounds is broken by the CDW gap appearing below $T_{\mathrm{CDW} 1}$. The drop of conductivity is much larger along the $a$ axis perpendicular to the CDW wave vector, leading to a strong in-plane conductivity anisotropy (see Fig. 1). We explain this effect by the direct calculation of the two components of conductivity for the electron dispersion with a momentum-dependent CDW gap as determined by ARPES . The CDW gap covers the FS parts where the mean square electron velocity along the $a$ axis is larger than along the $c$ axis, which leads to the conductivity anisotropy. This conductivity anisotropy can be used for an estimate of the electron dispersion parameter $t_{\perp}$. We also show that the electrical anisotropy is modified when the low- $T$ CDW occurs below $T_{\mathrm{CDW} 2}$, which can result from interplay between the two collective states.

We acknowledge $\mathrm{O}$. Leynaud for the $\mathrm{x}$-ray determination of $a$ and $c$ axis and T.F. Rosenbaum for sending us the preprint of Ref. [13] before publication. The work was supported by the Russian Foundation for Basic Research (Grants No. 11-02-01379-a and No. 13-02-00178-a), by SIMTECH Program (Grant No. 246937), and partially performed in the CNRS-RAS Associated International Laboratory between Institut Neel and IRE "Physical properties of coherent electronic states in coherent matter."

*Corresponding author.aasinch@mail.ru

[1] Z. Sun, Q. Wang, A. V. Federov, H. Zheng, J. F. Mitchell, and D. S. Dessau, Proc. Natl. Acad. Sci. U.S.A. 108, 11799 (2011).

[2] H. Seo, J. Phys. Soc. Jpn. 69, 805 (2000).

[3] S. A. Kivelson, I. P. Bindloss, E. Fradkin, V. Oganesyan, J. M. Tranquanda, A. Kapiltulnic, and C. Howald, Rev. Mod. Phys. 75, 1201 (2003).

[4] M. Vojta, Adv. Phys. 58, 699 (2009).

[5] E. DiMasi, M. C. Aronson, J. F. Mansfield, B. Foran, and S. Lee, Phys. Rev. B 52, 14516 (1995).

[6] V. Brouet, W. L. Yang, X. J. Zhou, Z. Hussain, R. G. Moore, R. He, D. H. Lu, Z. X. Shen, J. Laverock, S. B. Dugdale, N. Ru, and I. R. Fisher, Phys. Rev. B 77, 235104 (2008).
[7] N. Ru, C. L. Condron, G. Y. Margulis, K. Y. Shin, J. Laverock, S. B. Dugdale, M. F. Toney, and I. R. Fisher, Phys. Rev. B 77, 035114 (2008).

[8] M. Lavagnini, H.-M. Eiter, L. Tassini, B. Muschler, R. Hackl, R. Monnier, J.-H. Chu, I. R. Fisher, and L. Degiorgi, Phys. Rev. B 81, 081101(R) (2010).

[9] H. Yao, J. A. Robertson, E.-A. Kim, and S. A. Kivelson, Phys. Rev. B 74, 245126 (2006).

[10] D. LeBoeuf, S. Krämer, W. N. Hardy, R. Liang, D. A. Bonn, and C. Proust, Nat. Phys. 9, 79 (2013).

[11] N. Ru and I. R. Fisher, Phys. Rev. B 73, 033101 (2006).

[12] A. Fang, N. Ru, I. R. Fisher, and A. Kapitulnik, Phys. Rev. Lett. 99, 046401 (2007).

[13] A. Banerjee, Y. Feng, D. M. Silevitch, J. Wang, J. C. Lang, H.-H. Kuo, I. R. Fisher, and T. F. Rosenbaum, Phys. Rev. B 87, 155131 (2013).

[14] A. A. Sinchenko, P. Lejay, and P. Monceau, Phys. Rev. B 85, 241104(R) (2012).

[15] H. C. Montgomery, J. Appl. Phys. 42, 2971 (1971).

[16] B. F. Logan, S. O. Rice, and R. F. Wick, J. Appl. Phys. 42, 2975 (1971).

[17] N. P. Ong and J. W. Brill, Phys. Rev. B 18, 5265 (1978).

[18] A. A. Abrikosov, Fundamentals of the Theory of Metals (North-Holland, Amsterdam, 1988).

[19] The dependence of $\varepsilon(\boldsymbol{k})$ and $v_{i}^{2}(\boldsymbol{k})$ on the interlayer momentum $k_{z}$ is negligible because of strong quasi-2D anisotropy. Hence, the summation over interplane component $k_{z}$ in Eq. (1) is trivial and gives $1 / d$, where $d$ is the interlayer spacing.

[20] A. Kikuchi, J. Phys. Soc. Jpn. 67, 1308 (1998).

[21] F. Schmitt, P. S. Kirchmann, U. Bovensiepen, R. G. Moore, J-H. Chu, D. H. Lu, L. Rettig, M. Wolf, I. R. Fisher, and Z-X. Shen, New J. Phys. 13, 063022 (2011).

[22] The integration over the momentum at $T \ll E_{F}$ is performed in a standard way. The in-plane momentum integration is factorized into parallel and perpendicular to FS components: $d^{2} k=d k_{\|} d k_{\perp}=d k_{\|} d \epsilon / v_{F}$, where the Fermi velocity $v_{F}=$ $a \sqrt{t_{\|}^{2}\left\{1-\cos \left[\left(k_{x} \pm k_{y}\right) a\right]\right\}+t_{\perp}^{2}\left\{1-\cos \left[\left(k_{x} \mp k_{y}\right) a\right]\right\}}$ is independent of $\boldsymbol{k}$ up to the second-order terms in $t_{\perp} / t_{\|} \ll 1$, and $d k_{\|}=\sqrt{1+\left|d k_{y} / d k_{x}\right|_{F S}^{2}} d k_{x}$.

[23] Eq. (7) is an approximation of the mean-field dispersion in the presence of CDW with imperfect nesting, $E(\mathbf{k})=$ $[\varepsilon(\boldsymbol{k})+\varepsilon(\boldsymbol{k}-\boldsymbol{Q})] / 2 \pm \sqrt{[\varepsilon(\boldsymbol{k})-\varepsilon(\boldsymbol{k}-\boldsymbol{Q})]^{2} / 4+\Delta^{2}(T, \boldsymbol{k})}$, where $Q$ is the CDW wave vector.

[24] R. G. Moore, V. Brouet, R. He, D. H. Lu, N. Ru, J.-H. Chu, I. R. Fisher, and Z.-X. Shen, Phys. Rev. B 81, 073102 (2010).

[25] F. Pfuner, P. Lerch, J.-H. Chu, H.-H. Kuo, I. R. Fisher, and L. Degiorgi, Phys. Rev. B 81, 195110 (2010).

[26] P. D. Grigoriev, Phys. Rev. B 77, 224508 (2008).

[27] J. A. Wilson, F. J. Di Salvo, and S. Mahajan, Adv. Phys. 24, 117 (1975).

[28] P. Monceau, Adv. Phys. 61, 325 (2012).

[29] W. L. McMillan, Phys. Rev. B 16, 643 (1977).

[30] H-M. Eiter, M. Lavagnini, R. Hackl, E. A. Nowadnick, A. F. Kemper, T. P. Devereaux, J-H. Chu, J. G. Analytis, I. R. Fisher, and L. Degiorgi, Proc. Natl. Acad. Sci. U.S.A. 110, 64 (2013). 